\title{
THE APPROXIMATION PROPERTY DOES NOT IMPLY THE BOUNDED APPROXIMATION PROPERTY
}

\author{
T. FIGIEL AND W. B. JOHNSON ${ }^{1}$
}

\begin{abstract}
There is a Banach space which has the approximation property but fails the bounded approximation property. The space can be chosen to have separable conjugate, hence there is a nonnuclear operator on the space which has nuclear adjoint. This latter result solves a problem of Grothendieck [2].
\end{abstract}

I. Introduction. Let $(X,\|\cdot\|)$ be a Banach space. We show that if there is a constant $\lambda$ so that $(X,|\cdot|)$ has the $\lambda$-metric approximation property ( $\lambda$-m.a.p., in short) for each equivalent norm $|\cdot|$ on $X$, then $X^{*}$ has the bounded approximation property (b.a.p., in short). This result is used to construct an example of a Banach space which possesses the approximation property (a.p.) but fails the b.a.p.

For $\varepsilon, \lambda$ positive constants, we say that $X$ has the $(\varepsilon, \lambda)$-m.a.p. provided that, for each finite dimensional subspace $Z$ of $X$ and each $\delta>0$, there is a finite rank operator $T$ on $X$ so that $\|T\| \leqq \lambda+\delta$ and $\|T z-z\| \leqq(\varepsilon+\delta)\|z\|$ for each $z \in Z$. An intermediate step in our construction is that if $X$ has the $(\varepsilon, \lambda)$-m.a.p. for some $\varepsilon, 0<\varepsilon<1$, then $X$ has the $\lambda(1-\varepsilon)^{-1}$-m.a.p.

We use the standard notation in Banach space theory. Let us only recall the types of approximation conditions a Banach space $X$ may satisfy. $X$ has the a.p. if for each compact subset $K$ of $X$ and $\varepsilon>0$, there is a finite rank operator (=bounded, linear operator) $T$ on $X$ so that $\|T k-k\| \leqq \varepsilon$ for each $k \in K$. If always $T$ can be chosen with $\|T\| \leqq \lambda$ then $X$ is said to have the $\lambda$-m.a.p. A space which has the $\lambda$-m.a.p. for some $\lambda$ is said to have the b.a.p. For equivalent formulations of these definitions (which we use without further reference) the reader is referred to [2] and [4].

We wish to thank Professor A. Pełczyński for a revision of an earlier incorrect proof of the main result. Pelczyński's description [6] led us to the proof presented here.

Received by the editors January 18, 1973 and, in revised form, February 23, 1973. AMS (MOS) subject classifications (1970). Primary 46B99, 47B10; Secondary $41 \mathrm{~A} 65$.

Key words and phrases. Approximation property, nuclear operators.

1 The second author was supported in part by NSF GP-33578.

(c) American Mathematical Society 1973 
II. Implications among approximation conditions. Given a Banach space $(X,\|\cdot\|)$, let $\mathscr{A}$ be the family of equivalent norms, $|\cdot|$, on $X$ whose dual norms on $X^{*}$ are of the form $\left|x^{*}\right|=\left\|x^{*}\right\|+M d\left(x^{*}, Z\right)$. Here $M$ ranges over positive constants, $Z$ ranges over finite dimensional subspaces of $X^{*}$, and $d\left(x^{*}, Z\right) \equiv \inf \left\{\left\|x^{*}-z\right\|: z \in Z\right\}$ is the $\|\cdot\|$-distance of $x^{*}$ to $Z$. Since finite dimensional subspaces of $X^{*}$ are weak* closed, it is evident that each such norm on $X^{*}$ is the dual of an equivalent norm on $X$.

Proposition 1. Suppose that $(X,|\cdot|)$ has the $\lambda$-m.a.p. for each norm, $|\cdot|$, in $\mathscr{A}$. Let $0<\varepsilon<1$. Then $\left(X^{*},\|\cdot\|\right)$ has the $\left(\varepsilon, \lambda\left[1+2 \varepsilon^{-1} \lambda\right]\right)-$ m.a.p.

Proof. Suppose that $Z$ is a finite dimensional subspace of $X^{*}$. Let $\beta>\lambda$ and $\delta>0$. Define $|\cdot|$ on $X^{*}$ by $\left|x^{*}\right|=\left\|x^{*}\right\|+2 \varepsilon^{-1} \beta d\left(x^{*}, Z\right)$.

Pick a finite dimensional subspace $Y$ of $X$ such that for each $z \in Z$, $\|z\| \leqq(1+\delta) \sup \{z(y): y \in Y,\|y\| \leqq 1\}$. Since $(X,|\cdot|)$ has the $\lambda$-m.a.p., there is a finite rank operator $T$ on $X$ so that $T y=y$ for $y \in Y$ and $|T| \leqq \beta$.

We have, for $x^{*} \in X^{*}$,

$$
\left\|T^{*} x^{*}\right\|+2 \varepsilon^{-1} \beta d\left(T^{*} x^{*}, Z\right) \leqq \beta\left[\left\|x^{*}\right\|+2 \varepsilon^{-1} \beta d\left(x^{*}, Z\right)\right] .
$$

Hence $\left\|T^{*} x^{*}\right\| \leqq \beta\left(1+2 \varepsilon^{-1} \beta\right)\left\|x^{*}\right\|$ whence $\|T\| \leqq \beta\left(1+2 \varepsilon^{-1} \beta\right)$. Now for $z \in Z, 2 \varepsilon^{-1} \beta d\left(T^{*} z, Z\right) \leqq \beta\|z\|$, so there exists $w \in Z$ satisfying $\left\|T^{*} z-w\right\| \leqq$ $\frac{1}{2} \varepsilon\|z\|$. But for $y \in Y,\left(T^{*} z\right) y=z(T y)=z(y)$, and thus $\sup \{|z(y)-w(y)|$ : $y \in Y,\|y\| \leqq 1\} \leqq \frac{1}{2} \varepsilon\|z\|$. Therefore $\|z-w\| \leqq \frac{1}{2} \varepsilon(1+\delta)\|z\|$, from which it follows that

$$
\left\|T^{*} z-z\right\| \leqq\left[\frac{1}{2} \varepsilon(1+\delta)+\frac{1}{2} \varepsilon\right]\|z\| \leqq(1+\delta) \varepsilon\|z\| .
$$

Since $\delta>0, \beta>\lambda$ are arbitrary, the conclusion follows.

Proposition 2. Suppose $(X,\|\cdot\|)$ has the $(\varepsilon, \lambda)$-m.a.p. with $\varepsilon<1$. Then $X$ has the $(1-\varepsilon)^{-1} \lambda$-m.a.p.

Proof. We thank Professor W. J. Davis for the proof given here. Davis' proof is rather more revealing than proofs discovered by us.

Suppose $Z$ is a finite dimensional subspace of $X$. Let $0<\varepsilon<\delta<1$ and $\beta>\lambda$.

Construct by induction finite rank operators $T_{n}$ on $X$ so that

$$
\left\|T_{1} z-z\right\| \leqq \delta\|z\| \quad \text { for } z \in Z, \quad\left\|T_{n+1} x-x\right\| \leqq \delta\|x\|
$$

for $x \in \operatorname{span} Z \cup T_{n} X \cup T_{n-1} X \cup \cdots \cup T_{1} X$, and $\left\|T_{n}\right\| \leqq \beta$.

Define $S_{n}$ by $I-S_{n}=\left(I-T_{n}\right)\left(I-T_{n-1}\right) \cdots\left(I-T_{1}\right)$. Then for $z \in Z$, $\left\|\left(I-S_{n}\right) z\right\| \leqq \delta^{n}\|z\|$. Also,

$$
\begin{aligned}
S_{n}=\left(I-T_{n}\right)\left(I-T_{n-1}\right) & \cdots\left(I-T_{2}\right) T_{1}+\left(I-T_{n}\right)\left(I-T_{n-1}\right) \cdots \\
& \left(I-T_{3}\right) T_{2}+\cdots+\left(I-T_{n}\right) T_{n-1}+T_{n},
\end{aligned}
$$

so that $\left\|S_{n}\right\| \leqq \delta^{n-1} \beta+\cdots+\delta \beta+\beta<(1-\delta)^{-1} \beta$. 
Hence $X$ has the $\left(\tau,(1-\delta)^{-1} \beta\right)$-m.a.p. for each $\tau>0, \delta>\varepsilon$ and $\beta>\lambda$, whence $X$ has the $(1-\varepsilon)^{-1} \lambda$-m.a.p.

Setting $\varepsilon=\frac{1}{2}$ in the above two propositions yields:

THeOREM 1. If, for each $|\cdot|$ in $\mathscr{A},(X,|\cdot|)$ has the $\lambda$-m.a.p., then $X^{*}$ has the $2 \lambda(1+4 \lambda)$-m.a.p.

Remark 1. If $X^{*}$ has the $\lambda$-m.a.p. then for each $|\cdot|$ in $\mathscr{A},\left(X^{*},|\cdot|\right)$ has the $\lambda$-m.a.p. (hence also $(X,|\cdot|)$ has the $\lambda$-m.a.p.). For if $\left|x^{*}\right|=\left\|x^{*}\right\|+$ $M d\left(x^{*}, Z\right), Y$ is a finite dimensional subspace of $X^{*}$, and $\varepsilon>0$, then there is a finite rank operator $T$ on $X^{*}$ so that $\|T\| \leqq \lambda+\varepsilon$ and $T x^{*}=x^{*}$ for $x^{*} \in \operatorname{span} Y \cup Z$. Since $T$ is the identity on $Z,|T| \leqq\|T\| \leqq \lambda+\varepsilon$.

Remark 2. It is known [3, Theorem 4] that if $(X,|\cdot|)$ has the 1-m.a.p. for each $|\cdot|$ in $\mathscr{A}$, then $X^{*}$ has the 1-m.a.p. We do not know whether a similar result is true with " 1 " replaced by " $\lambda$ ". It may even be true that if $X^{*}$ has the b.a.p., then $X^{*}$ also has the 1-m.a.p. This is the case when $X^{*}$ is separable [4, Remark 4.11].

EXAMPLE. There is a Banach space which has the a.p. but fails the b.a.p.

Proof. Of course, we need the important result of Enflo [1] that there is a Banach space which fails the a.p. Lindenstrauss [5] (see [3] for a specific example) had shown that a consequence of this is the existence of a Banach space $X$ which possesses the 1-m.a.p., but whose conjugate fails the a.p. By Theorem 1 , there is a sequence $\left(|\cdot|_{n}\right)$ of equivalent norms on this $X$ so that $\left(X,|\cdot|_{n}\right)$ fails the $n$-m.a.p. Thus $\left(\Sigma\left(X,|\cdot|_{n}\right)\right)_{l_{2}}$ fails the b.a.p. but possesses the a.p.

Note that $\left(\Sigma\left(X,|\cdot|_{n}\right)\right)_{l_{2}}$ can be chosen to have separable conjugate, since Lindenstrauss' construction can yield an $X$ with $X^{*}$ separable.

III. Nonnuclear operators with nuclear adjoints. The example constructed in $\S I$ justifies the following proposition.

Proposition 3. If a Banach space $X$ has the a.p. but fails the b.a.p., and $X^{*}$ is separable, then there is a nonnuclear operator $T$ on $X$ such that $T^{*}$ is nuclear.

Since the proof is an almost immediate consequence of results of [2], we give only some indications.

Let $N(X)$ denote the space of nuclear operators on $X$ [2, Definition 4] and $L_{0}(X)$ the space of finite rank operators on $X$. Since the weak*continuous nuclear operators form a closed subset of $N\left(X^{*}\right)$, it is enough to show that $\left\{T^{*}: T \in N(X)\right\}$ is not closed in $N\left(X^{*}\right)$.

Consider the natural mappings $X^{*} \hat{\otimes} X^{\underline{\varphi}} \rightarrow N(X)^{\stackrel{x}{\rightarrow}}\left[L_{0}(X)\right]^{*} \stackrel{\underline{\Psi}}{\leftarrow} N\left(X^{*}\right)$. Here $\chi(T) S=\operatorname{trace} S T$ and $\psi(T) S=\operatorname{trace}\left(T S^{*}\right)$. 
Observe that

(i) $\varphi$ is an isometry onto, because $X$ has the a.p. (cf. [2, Proposition 35, $\left.\left.A \Rightarrow B_{1}\right]\right)$.

(ii) $\chi \varphi$ is not an isomorphic embedding, for otherwise (cf. [2, Proposition 39, proof of $B_{1} \Rightarrow A_{1}$ ]) $X$ would have the b.a.p.

(iii) $\psi$ is an isometry onto. For given $F \in L_{0}(X)^{*}$, consider the factorization (cf. [2, Proposition 27, (a) $\Rightarrow$ (d)]) $X^{*} \rightarrow L_{\infty} \rightarrow L_{1} \rightarrow X^{*}$ of the operator induced on $X^{*}$ by $F . X^{*}$ is separable, so the Dunford-Pettis theorem yields (cf. [2, Lemma 9]) that $L_{\infty} \rightarrow L_{1} \rightarrow X^{*}$ is nuclear.

Since (i), (ii), and (iii) imply that the range of $\psi^{-1} \chi$ is not closed, it only remains to observe that $\psi^{-1} \chi(T)=T^{*}$ for each $T \in N(X)$.

\section{REFERENCES}

1. P. Enflo, A counter example to the approximation problem, Acta Math. (to appear).

2. A. Grothendieck, Produits tensoriels topologiques et espaces nucléaires, Mem. Amer. Math. Soc. No. 16 (1955). MR 17, 763.

3. W. B. Johnson, A complementably universal conjugate Banach space and its relation to the approximation problem, Israel J. Math. 13 (1972), 301-310.

4. W. B. Johnson, H. P. Rosenthal and M. Zippin, On bases, finite dimensional decompositions, and weaker structures in Banach spaces, Israel J. Math. 9 (1971), 488-506. MR 43 \#6702.

5. J. Lindenstrauss, On James' paper 'Separable conjugate spaces,' Israel J. Math. 9 (1971), 279-284. MR 43 \#5289.

6. A. Pełczyński, Collected letters (unpublished).

Institute of Mathematics, Polish Academy of Sciences, Warsaw, Poland

Department of Mathematics, Ohio State University, Columbus, Ohio 43210 (Current address of W. B. Johnson)

Current address (T. Figiel): Department of Mathematics, Ohio State University, Columbus, Ohio 43210 